\title{
Combination antiretroviral therapy improves cognitive performance and functional connectivity in treatment-naïve HIV-infected individuals
}

\author{
Yuchuan Zhuang ${ }^{1} \cdot$ Xing Qiu $^{2} \cdot$ Lu Wang $^{2}$ • Qing Ma ${ }^{3}$ - Mark Mapstone ${ }^{4}$. \\ Amneris Luque $^{5} \cdot$ Miriam Weber $^{6} \cdot$ Madalina Tivarus $^{7} \cdot$ Eric Miller $^{8}$. \\ Roberto C. Arduino ${ }^{9}$ - Jianhui Zhong ${ }^{7}$ • Giovanni Schifitto 6,7
}

Received: 27 February 2017 / Revised: 20 May 2017 / Accepted: 10 July 2017 / Published online: 8 August 2017

(C) The Author(s) 2017. This article is an open access publication

\begin{abstract}
Our study aimed to investigate the short-term effect of combination antiretroviral therapy (cART) on cognitive performance and functional and structural connectivity and their relationship to plasma levels of antiretroviral (ARV) drugs. Seventeen ARV treatment-naïve HIV-infected individuals (baseline mean CD4 cell count, $479 \pm 48$ cells $/ \mathrm{mm}^{3}$ ) were age matched with 17 HIV-uninfected individuals. All subjects underwent a detailed neurocognitive and functional assessment and magnetic resonance imaging. HIV-infected subjects were scanned before starting cART and 12 weeks after initiation of treatment. Uninfected subjects were assessed once at baseline. Functional connectivity (FC) was assessed within the default mode network while structural connectivity was assessed by voxel-wise analysis using tract-based spatial statistics (TBSS) and probabilistic tractography within the DMN. Tenofovir and emtricitabine blood concentration were measured at week 12 of cART. Prior to cART, HIV-infected individuals had significantly lower cognitive performance than
\end{abstract}

Electronic supplementary material The online version of this article (doi:10.1007/s13365-017-0553-9) contains supplementary material, which is available to authorized users.

Giovanni Schifitto

Giovanni_Schifitto@URMC.Rochester.edu

1 Department of Electrical and Computer Engineering, University of Rochester, Rochester, NY, USA

2 Department of Biostatistics and Computational Biology, School of Medicine and Dentistry, University of Rochester Medical Center, Rochester, NY, USA

3 Department of Pharmacy Practice, University at Buffalo, Buffalo, NY, USA

4 Department of Neurology, University of California, Irvine School of Medicine, Irvine, CA, USA control subjects as measured by the total Z-score from the neuropsychological tests assessing six cognitive domains $(p=0.020)$. After 12 weeks of cART treatment, there remained only a weak cognitive difference between HIV-infected and HIV-uninfected subjects $(p=0.057)$. Mean FC was lower in HIV-infected individuals compared with those uninfected ( $p=0.008)$, but FC differences became non-significant after treatment $(p=0.197)$. There were no differences in DTI metrics between HIV-infected and HIV-uninfected individuals using the TBSS approach and limited evidence of decreased structural connectivity within the DMN in HIV-infected individuals. Tenofovir and emtricitabine plasma concentrations did not correlate with either cognitive performance or imaging metrics. Conclusions: Twelve weeks of cART improves cognitive performance and functional connectivity in ARV treatment-naïve HIV-infected individuals with relatively preserved immune function. Longer periods of observation are necessary to assess whether this effect is maintained.
Department of Internal Medicine, University of Texas Southwestern Medical Center, Dallas, TX, USA

6 Department of Neurology, School of Medicine and Dentistry, University of Rochester Medical Center, Rochester, NY, USA

7 Department of Imaging Sciences, School of Medicine and Dentistry, University of Rochester Medical Center, 601 Elmwood Ave, Box 673, Rochester, NY 14642, USA

8 Department of Psychiatry and Bio-behavioral Sciences, University of California, Los Angeles, CA, USA

9 Division of Infectious Diseases, Department of Internal Medicine, The University of Texas Health Science Center at Houston, Houston, TX, USA 
Keywords HIV infection · Combination antiretroviral therapy $\cdot$ Functional magnetic resonance imaging $\cdot$ Diffusion tensor imaging $\cdot$ Cognitive function

\section{Introduction}

HIV infection is associated with injury of the central nervous system (CNS) (Navia et al. 1986). The clinical manifestations of CNS injury vary from a mild decrease in cognitive performance without associated functional deficits to dementia (Antinori et al. 2007). Neuroimaging biomarkers provide an opportunity to investigate CNS injury especially at the time when clinical changes may be small or silent (Zhu et al. 2013). Most of the recent literature on HIV-associated cognitive impairment that has included neuroimaging is derived from patient with long established HIV infection and on stable combination antiretroviral treatment (cART) (Hua et al. 2013). It has been reassuring that the cognitive deficits of those on cART are often mild and the neuroimaging abnormalities are limited. However, we still have limited understanding of the causes for the persistence of cognitive impairment in HIVinfected individuals who have been virologically suppressed for years. One plausible explanation is that irreversible CNS damage occurs very early in HIV infection which, when limited, causes subtle cognitive changes measurable to some degree with extensive neuropsychological tests. In this case, effective cART would prevent progression of CNS injury but not reverse previous damage (Tozzi et al. 2005). Previous reports on cognitive improvement (not necessarily resolution) in patients with dementia after starting cART support this possibility (Chang et al. 1999). However, it is also possible that long-term treatment with cART may also induce neurotoxicity (Robertson et al. 2010). A bimodal response could be occurring where uncontrolled viral infection and associated inflammation would greatly benefit from cART resulting in cognitive improvement and decreased abnormalities in imaging metrics (Chang et al. 1999). To study the early time course progression of CNS injury in HIV infection, we focused on the default mode network (DMN) as the major region to investigate because it has been widely studied and reported to be affected by HIV infection (Becker et al. 2013; Herting et al. 2015; Ortega et al. 2015; Thomas et al. 2013). Here, we report our initial findings, assessing whether cognitive, functional, and structural brain connectivity were altered prior to the initiation of cART and whether there were measurable changes after 12 weeks of CART treatment.

\section{Materials and methods}

All subjects, who consented to participate in the study, underwent a comprehensive clinical, laboratory (chemistry, hematology, and urine analysis), neurocognitive, and neuroimaging evaluation. HIV-infected individuals were assessed before and 12 weeks after starting cART, while HIVuninfected controls were assessed only at baseline.

Inclusion criteria All participants were $\geq 18$ years of age and antiretroviral (ARV) treatment-naïve prior to enrollment. They met the following laboratory parameters within 30 days of baseline evaluation: hemoglobin $\geq 9.0 \mathrm{~g} / \mathrm{dL}$, serum creatinine $\leq 2 \times$ ULN, AST (SGOT), ALT (SGPT), and alkaline phosphatase $\leq 2 \times$ upper limit of normal. Subjects were instructed to avoid smoking and use of caffeinated drinks for at least $2 \mathrm{~h}$ prior to the scheduled imaging section.

Exclusion criteria Participants with severe premorbid or comorbid psychiatric disorders were excluded. Subjects with mild or stable depression including those on stable antidepressant therapy were eligible for this study. Additional exclusion criteria were stroke, head trauma resulting in loss of consciousness $>30 \mathrm{~min}$, multiple sclerosis, brain infections (except for HIV-1), and any space-occupying brain lesions requiring acute or chronic therapy. Dementia, as established by HAND definition (Antinori et al. 2007), was exclusionary. Subjects meeting criteria for HIV-associated mild neurocognitive disorder (MND) or HIV-associated asymptomatic neurocognitive impairment (ANI) were eligible to participate. Active alcohol and drug abuse (urine toxicology was done at each visit) within 6 months of study entry and conditions such as claustrophobia or metallic implant that prevented MRI scanning were exclusionary.

Neuropsychological tests The neurocognitive evaluation was performed by trained staff and supervised by a neuropsychologist and included tests of executive function (Trailmaking Test Part B, Stroop Interference Task), speed of information processing (Symbol Digit Modalities Test and Stroop Color Naming), attention and working memory (CalCAP(CRT4) and WAIS-III Letter-Number Sequencing), learning (Rey Auditory Verbal Learning Test AVLT (trials 1-5), Rey Complex Figure Test Immediate Recall), memory (Rey Auditory Verbal Learning Test RAVLT Delayed Recall, Rey Complex Figure Test Delayed Recall), and motor (Grooved Pegboard, the left and right hands). An estimate of premorbid intellectual functioning ability was obtained via WRAT-4 Reading. The total composite Z-score was the primary cognitive outcome and was created from the linear combination of the Z-scores of the six cognitive domains measured (executive function, speed of information processing, attention and working memory, learning, memory, and motor). HAND diagnoses were determined for each participant according to the Frascati criteria (Antinori et al. 2007). 
MRI data acquisition MRI was performed on a 3T Siemens MAGNETOM Trio MRI scanner equipped with a 32-channel head coil at the Rochester Center for Brain Imaging. A T1weighted three-dimensional magnetization-prepared rapid acquisition gradient echo (MPRAGE, repetition time (TR)/inversion time $(\mathrm{TI}) /$ echo time $(\mathrm{TE})=2530 / 1100 / 3.44 \mathrm{~ms}$, voxel size $=1.0 \times 1.0 \times 1.0 \mathrm{~mm}^{3}$, flip angle $=78^{\circ}$, bandwidth $=190 \mathrm{~Hz} /$ pixel) was acquired. DTI scans were acquired with the following parameters: $10 \mathrm{~b}=0 \mathrm{~s} / \mathrm{mm}^{2}$ images acquired increase signal to noise ratio; 60 diffusion weighting images which uniformly distributed with $\mathrm{b}=1000 \mathrm{~s} / \mathrm{mm}^{2}$; voxel size $=2 \times 2 \times 2 \mathrm{~mm}^{3}$; matrix size $=128 \times 128$. A double-echo gradient echo field map sequence was acquired with the same resolution as the DTI sequence and was used to correct for distortion caused by B0 inhomogeneity (Dietrich et al. 2008). The resting-state fMRI scans were acquired using a gradient echo-planar imaging (EPI) sequence $\left(\mathrm{TR}=2000 \mathrm{~ms}, \mathrm{TE}=30 \mathrm{~ms}\right.$, flip angle $=90^{\circ}$, voxel size $=4 \times 4 \times 4 \mathrm{~mm}^{3}$; matrix size $=64 \times 64,30$ axial slices, 150 time points). During the entire 5-min resting-state fMRI scanning, participants were instructed to keep their eyes open and avoid falling asleep.

Resting-state fMRI processing The first ten volumes were discarded for each subject to allow for stabilization of the magnetic field. Standard pre-processing steps were performed using Data Processing Assistant for Resting-State fMRI (DPARSF) (Chao-Gan and Yu-Feng 2010) and included the following: slice timing correction, motion correction, normalization, and spatial smoothing with Gaussian kernel (full width at half maximum $(\mathrm{FWHM})=4 \mathrm{~mm})$, linear trend removal, and band-pass filter $(0.01 \mathrm{~Hz}$ to $0.08 \mathrm{~Hz})$ to reduce the low-frequency drift and exclude physiological noise. We used maximum head motion displacement larger than $2 \mathrm{~mm}$ and head rotation greater than $2^{\circ}$ as exclusion criteria. No subjects were excluded in this cohort. Each individual's fMRI images were normalized to Montreal Neurological Institute (MNI) standard space. To minimize head motion effect and nonneuronal noise, nuisance covariates including six head motion parameters, global mean signal, white matter time series, and CSF time series were regressed out. After pre-processing, group independent component analysis (ICA) was performed to identify the default mode network (DMN) using Group ICA part of fMRI Toolbox (GIFT, http://mialab.mrn.org/software/ gift/index.html). All subjects' resting-state fMRI data were included in a single group ICA analysis. Data were reduced through principal component analysis (PCA) (Calhoun et al. 2001; Erhardt et al. 2011) in two stages: one at the single subject level and a second one at the group level. Following data reduction, the multi-subject ICA was performed to identify the independent component using Infomax algorithm (Bell and Sejnowski 1995). GICA-based back-reconstruction methods were used to reconstruct single-subject components.
This resulted in 20 independent component (IC) spatial maps for each subject.

The single DMN component was firstly identified by visual inspection. The one-sample $t$ test was used to test the significance of the selected DMN component that resulted in a tmap. Only the posterior DMN component which includes the posterior cingulate cortex (PCC) and the inferior parietal cortex (IPC) were included after using $p<0.0001$ with FDR correction.

The average time series of all the voxels in the DMN was calculated and correlated with each voxel's own time series within DMN, to calculate within-network functional connectivity (Supekar et al. 2010). Fisher's r-to-z transformation was applied to the functional connectivity for each individual.

\section{Diffusion tensor imaging processing}

The first ten $b=0$ images and 60 diffusion-weighted images were firstly corrected for motion and eddy-current-induced distortion, using eddy correct tool in FSL (Smith et al. 2004; Woolrich et al. 2009; Jenkinson et al. 2012). The ten $b=0$ images were averaged as a b0 image, which was then used to register DTI data to T1 structural image using boundary-based registration (BBR) (Greve and Fischl 2009) in FSL. Brain extraction was performed on the averaged b0 image using BET (Smith 2002) in FSL. The averaged b0 image and 60 DWIs were used to calculate the tensor-derived scalar metrics including fractional anisotropy (FA), mean diffusivity (MD), radial diffusivity (RD), and axial diffusivity (AD) using DTIFIT in FSL. A brain mask was applied on each tensorderived scalar metric to create brain-extracted maps.

Voxel-wise analysis was performed using tract-based spatial statistics (TBSS) (Smith et al. 2006), part of FSL. Group comparisons were performed using the Randomize toolbox (Nichols and Holmes 2002; Winkler et al. 2014) in FSL, and the threshold-free cluster enhancement (TFCE) approach (Smith and Nichols 2009) was used for multiple comparison correction. We also performed probabilistic tractography within DMN using FSL. A DMN mask was manually selected from Group ICA analysis using resting-state $\mathrm{AMRI}$ : PCC served as seed region, and medial prefrontal cortex (mPFC), left- and right-inferior parietal cortex (IPC) served as target regions, respectively. The structural connectivity (SC) was calculated in two ways, the total number of voxel within the tracts, and the mean FA within the tracts. (See Supplemental material for details).

\section{Drug concentration measurement and pharmacokinetic analysis}

Plasma tenofovir (TFV) and emtricitabine (FTC) were measured using a newly developed and validated liquid 
chromatography with tandem mass spectrometry (LC-MS/ MS) method (Delahunty et al. 2009). TFV and FTC were chromatographically separated on the LC system, detected at the tandem MS system and calibrated within a linear range of 10-1000 ng/mL. The lower limit of quantitation for TFV and FTC was determined to be $10.0 \mathrm{ng} / \mathrm{mL}$, and assay precision was $<10 \%$. The study participants were taking ARV regimens that included tenofovir disoproxil fumarate (TDF) $300 \mathrm{mg}$ daily and FTC $200 \mathrm{mg}$ daily for at least 12 weeks. In order to estimate TFV and FTC exposure, population models were developed using the data from the AIDS Clinical Trials Group study A5202 (Beal 2001; Sax et al. 2009; Valade et al. 2014). TFV and FTC pharmacokinetics at the steady state was best described by a two-compartment linear mammillary model with first-order absorption with inter-individual variability on clearance. The exposure was indicated by the area under the plasma drug concentration-time curve (AUC, mg/L*h).

\section{Statistical analysis}

Data are presented as mean $\pm \mathrm{SE}$ throughout this manuscript. Comparisons between two independent groups were subjected to Wilcoxon rank-sum test. For comparing measurements collected from the same subject at two time points, Wilcoxon signed-rank test was used instead. Spearman correlation test was used for testing the dependence of two continuous variables. A $p$ value $<0.05$ was considered statistically significant.

All analyses were performed in R 3.2.0 (R Foundation for Statistical Computing, Vienna, Austria) and in SAS 9.3 (SAS Institute, Cary NC).

\section{Results}

Seventeen ARV treatment-naïve HIV-infected individuals were age matched with $17 \mathrm{HIV}$-uninfected individuals. HIVinfected individuals were scanned before starting cART, and, on average, 12 weeks after the initiation of ARV treatment. Demographics and baseline clinical characteristics are shown in Table 1. The groups were well balanced for age and education. The uninfected group was more likely to be female and White. At baseline, ten HIV-infected individuals had normal cognitive performance, six had ANI, and one had MND. Applying HAND classification to HIV-uninfected individuals, there was a similar proportion of cognitive impairment. However, when using the composite Z-score of all cognitive tests, HIV-infected individuals performed worse than HIVuninfected individuals (Table 2). The mean CD4 cell count and HIV RNA levels at baseline were $479 \pm 48$ cells $/ \mathrm{mm}^{3}$ and $5.08 \pm 4.64 \log _{10}$ copies $/ \mathrm{mL}$, respectively. The relatively high CD4 cell count in the HIV-infected individuals at baseline likely reflects a recent HIV infection; however, timing from exposure to HIV to diagnosis was not available. After
12 weeks, mean CD4 cell count and HIV RNA levels were $636 \pm 55$ cells $/ \mathrm{mm}^{3}$ and $899 \pm 861$ copies $/ \mathrm{mL}$, respectively.

Antiretroviral regimens prescribed included Complera (rilpivirine, FTC, TDF, $n=2$ ), Stribild (elvitegravir, cobicistat, FTC, TDF, $n=8$ ), Atripla (efavirenz, FTC, TDF, $n=3$ ), Truvada (FTC/TDF) plus Tivicay (dolutegravir, $n=1$ ), Truvada (FTC/TDF) plus Isentress (raltegravir, $n=1$ ), and Triumeq (dolutegravir, abacavir, lamivudine, $n=1$ ).

The results in Table 2 show that the mild cognitive deficits present at baseline in HIV-infected compared with HIVuninfected individuals, tended to improve in most cognitive domains, with exception of motor function, after 12 weeks of cART.

Functional connectivity (FC) was evaluated within the DMN. For these analyses, 16 HIV-infected and 16 HIVuninfected individuals were available at baseline and 14 HIV-infected individuals at 12 weeks. Mean FC at baseline was 0.588 in HIV-infected and 0.689 in those uninfected $(p$ value $=0.008$ ). After 12 weeks of cART, mean FC increased to 0.637 , and when compared with HIV-uninfected individuals, the difference was not significant ( $p$ value $=0.197$ ) Figure 1 shows the DMN in this population as determined using ICA and the mean FC in the DMN for each group of subjects and visits.

We further examined the relationship between FC and cognitive performance. FC was significantly correlated with total $\mathrm{Z}$-score in HIV-infected individuals at baseline (Spearman correlation $0.50, p$ value $=0.046$ ) (see Fig. 2) but was not significantly correlated with either HIV-infected after 12 weeks of cART or HIV uninfected. Other variables such as CD4 cell count, HIV RNA levels, and the duration of HIV infection were not significantly correlated with FC (Fig. 3).

We also assessed whether the FC and total Z-score correlated with plasma exposure of TFV and FTC. For both TFV and FTC, there was no significant correlation between FC and plasma exposure ( $p$ value $=0.73$ and 0.26 , respectively) and $\mathrm{Z}$-score and plasma exposure of these ARVs ( $p$ value $=0.58$ and 0.51 , respectively).

White matter microstructure abnormalities were assessed via diffusion tensor imaging and specifically via TBSS and probabilistic tractography within the DMN. No significant differences were found in TBSS analyses between HIVinfected and HIV-uninfected individuals nor in HIV-infected individuals before and after 12 weeks of cART. However, the results from the probabilistic tractography were inconclusive. While the total number of voxels from PCC to left-IPC was significantly greater in HIV-uninfected compared with HIVinfected both at baseline and 12 weeks of treatment, $p<0.01$ and $p<0.05$, respectively, the mean FA within the tract was not significant. In addition, we did not find significant differences in the other two pairs of tracts, in either number of voxels or mean FA. Lastly, there was no significant correlation between $\mathrm{SC}$ and FC. 
Table 1 Demographics and baseline clinical variables

\begin{tabular}{|c|c|c|c|}
\hline & HIV infected $(n=17)$ & HIV uninfected $(n=17)$ & $p$ value \\
\hline Age ((years) mean $\pm \mathrm{SE})$ & $32.6 \pm 2.9$ & $32.4 \pm 3.0$ & 0.956 \\
\hline Gender (M:F) & $17: 0$ & $7: 10$ & $<0.001$ \\
\hline Ethnicity (White:Black:other) & 8:9:0 & $12: 3: 2$ & 0.042 \\
\hline \multicolumn{4}{|l|}{ Education } \\
\hline $\begin{array}{l}\leq 12 \text { years } \\
>12 \text { years }\end{array}$ & $\begin{array}{l}4 \\
13\end{array}$ & $\begin{array}{l}3 \\
14\end{array}$ & 1 \\
\hline $\begin{array}{l}\text { HIV duration by patient report } \\
\text { at baseline ((months) median (range)) }\end{array}$ & $1(1,120)$ & NA & - \\
\hline $\begin{array}{l}\text { Baseline CD4 cell count } \\
\qquad\left(\left(\text { cells } / \mathrm{mm}^{3}\right) \text { mean } \pm \mathrm{SE}\right)\end{array}$ & $479.2 \pm 48.5$ & NA & - \\
\hline $\begin{array}{l}\text { Baseline HIV RNA levels } \\
\quad\left(\left(\log _{10} \text { unit) median (range) }\right)\right. \\
\text { HAND classification at baseline }\end{array}$ & $4.7(1.7,5.8)$ & NA & - \\
\hline $\begin{array}{l}\text { Normal } \\
\text { ANI }\end{array}$ & $\begin{array}{l}10 \\
6\end{array}$ & $\begin{array}{l}11 \\
5\end{array}$ & \multirow[t]{2}{*}{1} \\
\hline MND & 1 & 1 & \\
\hline
\end{tabular}

HAND HIV-Associated Neurocognitive Disorder, ANI Asymptomatic Neurocognitive Impairment, MND Mild Neurocognitive Disorder

\section{Discussion}

There is still ongoing debate on whether long-term use of cART may have deleterious effect on brain structure and function (Robertson et al. 2010; Cysique et al. 2009; Smurzynski et al. 2011; Marra et al. 2009). Neuroimaging has been used as a noninvasive tool to assess CNS injury in HIV-infected individuals. A few studies have assessed the impact of HIV infection and cART on cognitive performance and imaging metrics using fMRI or diffusion tensor imaging in ARV treatment-naïve individuals in a cross-sectional design (Ortega et al. 2015; Chang et al. 2008; Ragin et al. 2015). Our study adds to this literature by evaluating a longitudinal design and ARV regimens that reflects current standard of practice in the USA. We found significant differences in cognitive performance, as measured by the total composite Z-score created from combination of all neuropsychological tests used, and functional connectivity (FC) within DMN in ARV treatment-naïve HIV-infected compared with HIV-uninfected individuals that improved after 12 weeks of cART. Of note, neither baseline HIV RNA levels nor CD4 cell count correlated with either FC or cognitive performance.

A recent cross-sectional study by Ortega et al. (2015) analyzed cognitive performance and FC in three groups of individuals: HIV-uninfected, HIV-infected on cART, and HIV-infected not on cART. The group not on cART was a mix of never on cART or currently not taking cART. The investigators found that HIV-infected individuals had lower FC than HIVuninfected individuals in areas that included DMN, and this

Table 2 Cognitive performance by composite Z-score cognitive domain at baseline in HIV-uninfected individuals and in HIV-infected individuals before and after 12 weeks on cART

\begin{tabular}{|c|c|c|c|c|c|c|}
\hline Composite Z-score & $\begin{array}{l}\text { HIV-baseline } \\
(\text { mean } \pm \text { SE) }\end{array}$ & $\begin{array}{l}\text { HIV+ baseline } \\
(\text { mean } \pm \mathrm{SE})\end{array}$ & $\begin{array}{l}\text { HIV+ } 12 \text { weeks } \\
(\text { mean } \pm \text { SE })\end{array}$ & $\begin{array}{l}\text { HIV- vs. baseline } \\
\text { HIV+ }(p \text { value })^{\mathrm{a}}\end{array}$ & $\begin{array}{l}\text { HIV }+12 \text { weeks vs. } \\
\text { HIV- }(p \text { value })^{\mathrm{a}}\end{array}$ & $\begin{array}{l}\text { HIV+ baseline vs. } \\
12 \text { weeks }(p \text { value })^{\mathrm{b}}\end{array}$ \\
\hline Executive function & $0.30 \pm 0.28$ & $-0.24 \pm 0.23$ & $-0.06 \pm 0.21$ & 0.125 & 0.379 & 0.332 \\
\hline $\begin{array}{l}\text { Speed of } \\
\text { information } \\
\text { processing }\end{array}$ & $0.33 \pm 0.21$ & $-0.13 \pm 0.25$ & $-0.20 \pm 0.26$ & 0.179 & 0.158 & 1.000 \\
\hline Attention & $0.21 \pm 0.22$ & $-0.23 \pm 0.27$ & $0.02 \pm 0.23$ & 0.343 & 0.617 & 0.309 \\
\hline Learning & $0.09 \pm 0.22$ & $-0.13 \pm 0.22$ & $0.04 \pm 0.29$ & 0.502 & 0.667 & 0.404 \\
\hline Memory & $0.00 \pm 0.22$ & $-0.12 \pm 0.24$ & $0.11 \pm 0.27$ & 0.730 & 0.654 & 0.094 \\
\hline Motor & $0.53 \pm 0.22$ & $-0.29 \pm 0.26$ & $-0.24 \pm 0.21$ & 0.023 & 0.025 & 0.624 \\
\hline $\begin{array}{l}\text { Total composite } \\
\text { Z-score }\end{array}$ & $1.46 \pm 0.86$ & $-1.14 \pm 1.07$ & $-0.32 \pm 0.9$ & 0.020 & 0.057 & 0.174 \\
\hline
\end{tabular}

${ }^{\mathrm{a}}$ Wilcoxon rank-sum test

${ }^{\mathrm{b}}$ Wilcoxon signed-rank test

$p$-value $<0.05$ were in italics 
Fig. 1 Functional connectivity $(F C)$ within default mode network (DMN). Mean FC for HIV infected before treatment was 0.588 and increased to 0.637 after 12 weeks of cART, while the mean FC for HIV-uninfected group was $0.689 ; * p=0.008$
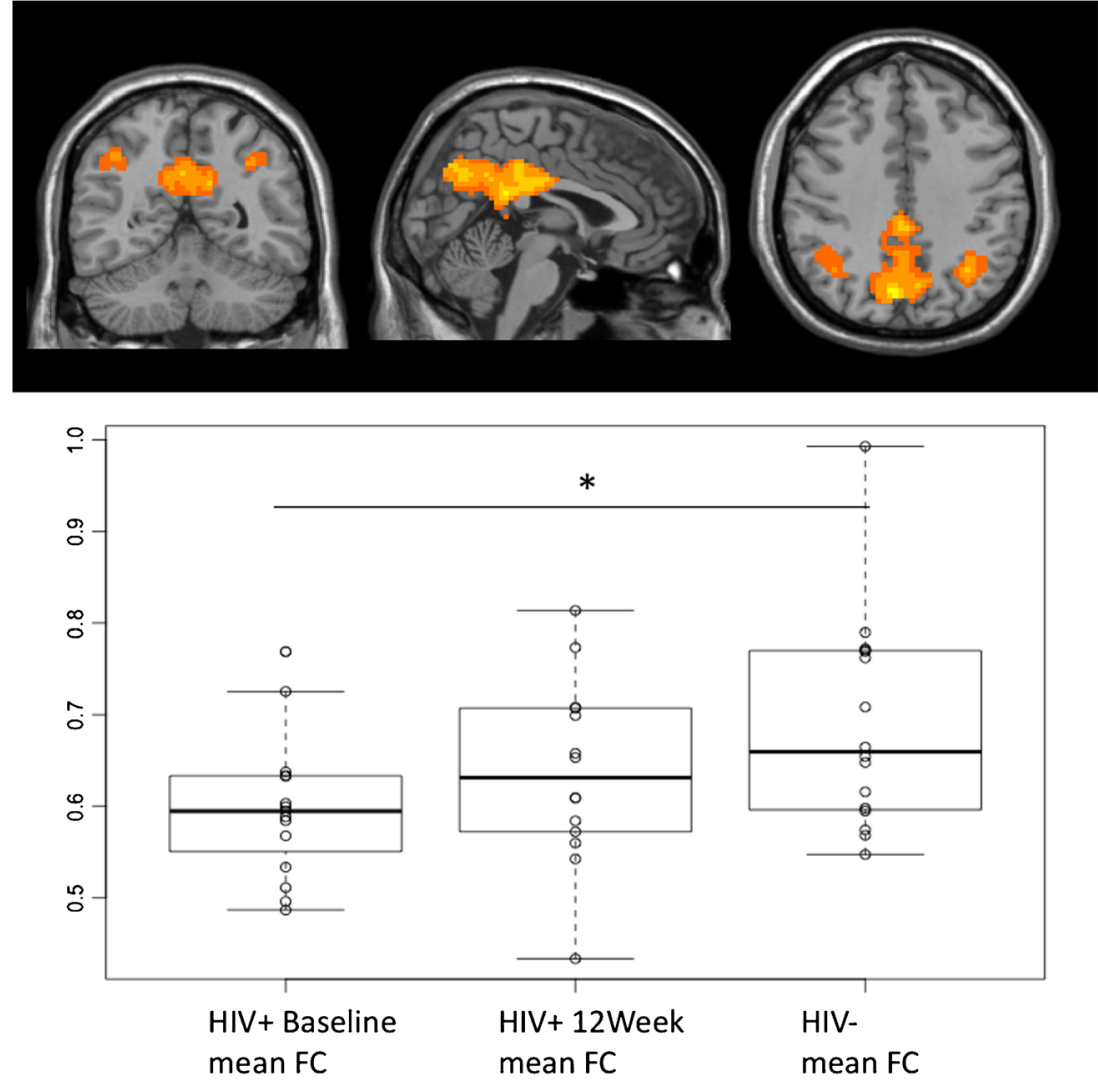

was even lower in those not on cART. Our results confirm these findings and provide longitudinal support that ARV treatment, at least in the short run, tends to normalize the FC. Importantly, we also show that cognitive performance correlated with FC before ARV treatment and that after treatment, improvement in cognitive performance rendered this correlation non-significant, similar to the observation in HIV-uninfected individuals.

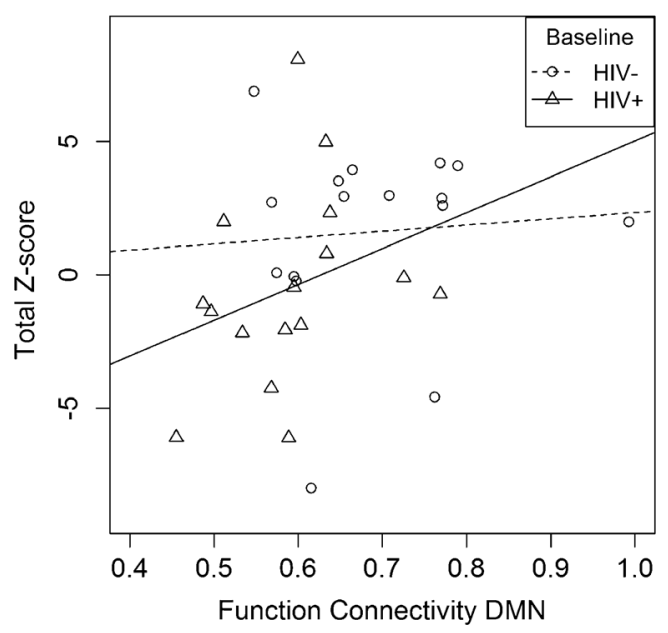

Fig. 2 Functional connectivity correlation with cognitive performance. FC was significantly correlated with total composite Z-score for HIVinfected individuals at baseline $(\rho=0.50, p$ value $=0.046)$ but not with HIV-uninfected individuals
While several studies have found some difference in FC, not necessarily in the same networks, between HIV-infected and HIV-uninfected individuals (Ortega et al. 2015; Thomas et al. 2013; Ipser et al. 2015; Wang et al. 2011), others have not (Janssen et al. 2016). However, these studies represent heterogeneous populations and analysis methods and thus are difficult to compare. Another study, for example, using a fMRI activation task, found that HIV-infected individuals on cART require greater brain activation than HIV-infected not on cART or HIV-uninfected, thus pointing to a possible deleterious effect of cART (Chang et al. 2008).

We and others have shown that HIV infection is associated with microstructural white matter abnormalities (Zhu et al. 2013; Ragin et al. 2005; Pfefferbaum et al. 2007; Pomara et al. 2001). These studies have included HIV-infected individuals with different degrees of immune suppression and comorbidities. The current study offers the opportunity to assess the effect of HIV viremia and cART on white matter in a longitudinal design in ARV treatment-naïve subjects with relative preserved immune function, comparing them very well with age-matched HIV-uninfected controls. We found no significant white matter microstructural differences (using TBSS) comparing HIV-infected with HIV-uninfected and HIV-infected before and after starting cART. These results differ from a recent cross-sectional study in patients recently infected, which found abnormalities in the corpus callosum 
Fig. 3 Correlations between plasma exposure of TFV, FTC, and $\mathrm{FC}$ and cognitive performance

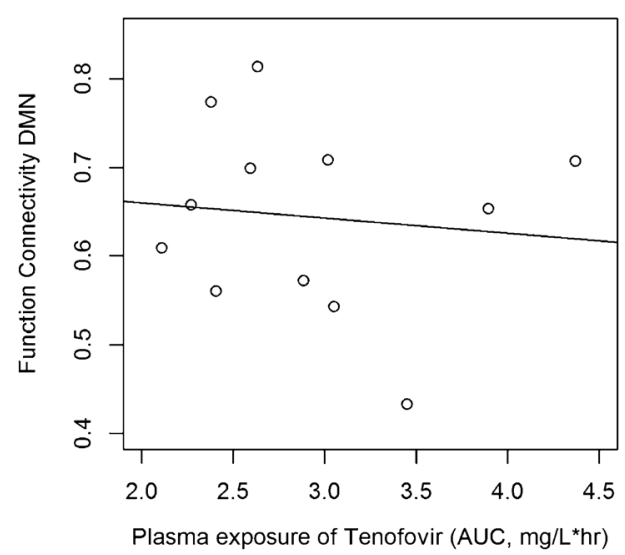

a

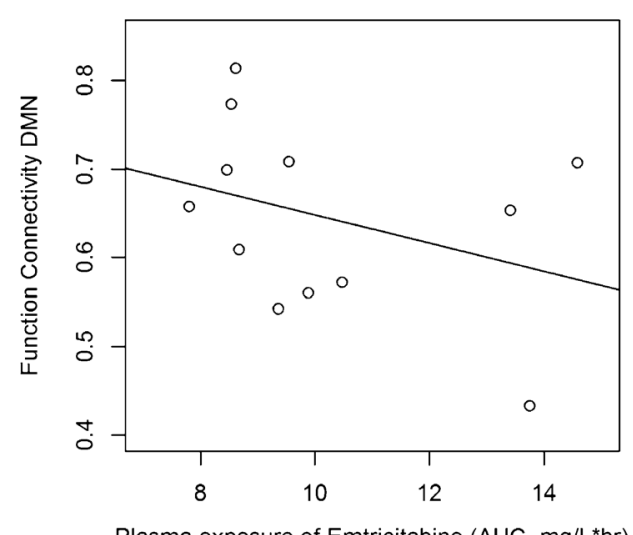

Plasma exposure of Emtricitabine (AUC, $\mathrm{mg} / \mathrm{L}^{*} \mathrm{hr}$ )

C
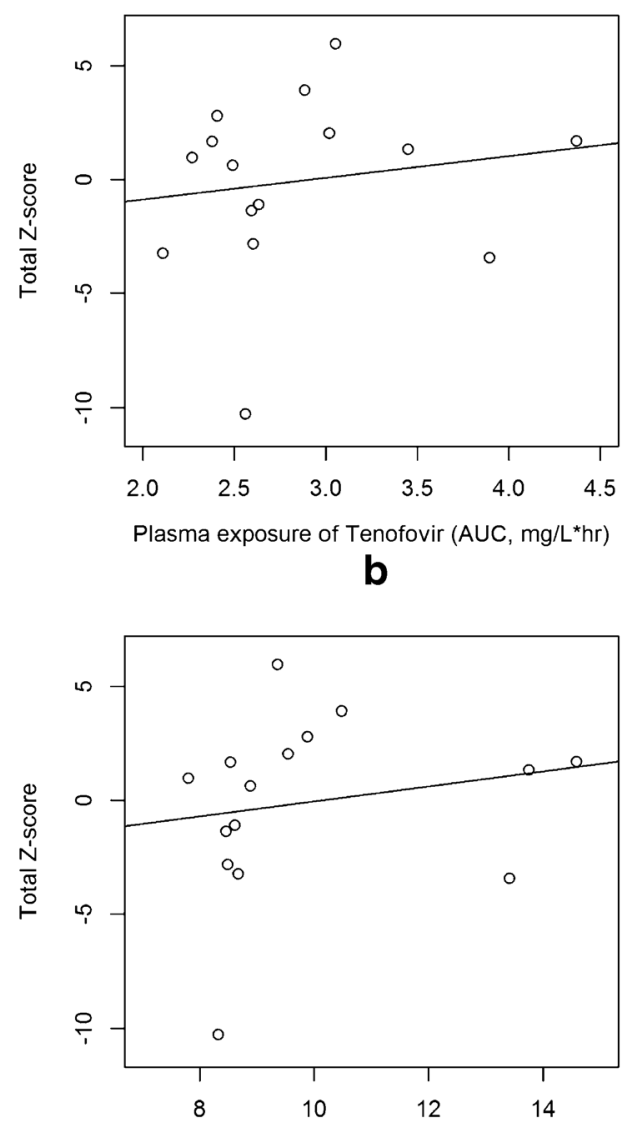

Plasma exposure of Emtricitabine (AUC, $\mathrm{mg} / \mathrm{L}^{*} \mathrm{hr}$ )

d
(Ragin et al. 2015). One possible explanation is that the patients in our study had a relatively well-preserved immune function and both HIV-infected and HIV-uninfected individuals were tightly age matched. Group differences in gender, as observed in our study, are less likely to affect DTI metrics while age has a large impact (Wu et al. 2011). Given the small sample size, variability in immune function and age may have a large effect on results.

In order to more directly compare functional and structural connectivity, we assessed structural connectivity within the DMN using probabilistic tractography. The results of these analyses were inconclusive as we found decreased connectivity, when measured by number of voxel, in HIV-infected individuals only in the tract connecting PCC to left IPC. However, mean FA within this tract, the PCC to right IPC, and PCC to medial prefrontal cingulate cortex did not differ between HIV-infected and HIV-uninfected individuals.

We did not find a significant correlation between the plasma exposures of TFV and FTC with both cognitive performance and FC. These results do not exclude potential longterm effect as suggested by a recent small study (Ma et al. 2015) where higher plasma concentrations of TFV and FTC were found in patients with declining cognitive performance. The distribution of these agents into the brain, particularly
TFV, is limited due to its anionic charge at physiological $\mathrm{pH}$ making it difficult to cross the blood-brain barrier. In fact, low TFV concentrations and accordingly high HIV RNA levels in cerebral spinal fluid (CSF) were reported in previous studies suggesting the CNS as a sanctuary for HIV viral replication and viral escape (Best et al. 2012; Calcagno et al. 2011).

Limitations in this study include small sample and short duration of the longitudinal follow-up. In addition, gender and ethnicity was significantly different in the two cohorts. However, these factors should have not significantly affected the neuroimaging results (Joel et al. 2015). Also, we cannot exclude that cognitive performance improved in the HIVinfected individuals secondary to a practice effect. The study design did not include a 12-week evaluation in the HIVuninfected group as we did not expect cognition to change within that period. However, the change observed in FC in HIV-infected individuals is unlikely to be explained by practice effect.

In summary, our results suggest that in fairly young ARV treatment-naïve individuals with relatively preserved immune function, there are signs of decreased functional connectivity that correlates with decreased cognitive performance. There was inconclusive evidence of white matter microstructural abnormalities suggesting that the process may be reversible 
if HIV infection is treated early. In this regard, the improvement in cognitive performance and FC after 12 weeks of cART is encouraging and would favor the current approach of treating HIV infection as early as possible. However, a longer longitudinal follow-up and larger study is necessary to assess whether the improvement observed is sustainable overtime or whether the potential neurotoxicity of cART will manifest.

Acknowledgments Alicia Tyrell, Andy Straw, and Jeanne HoldenWiltse provided data management. Study coordinators include Elizabeth Keller, Christine Hurley, Emily Cosimano, and Catherine Bunce.

Authors' contributions Yuchuan Zhuang is responsible for acquisition, analysis, and interpretation of the work, revising it critically for important intellectual content, final approval, and accountability of the version to be published. Xing Qiu and Qing Ma are responsible for design of the work, analysis, and interpretation of data for the work, revising it critically for important intellectual content, final approval, and accountability of the version to be published. Lu Wang is responsible for analysis and interpretation of data for the work, revising it critically for important intellectual content, final approval, and accountability of the version to be published. Amneris Luque, Mark Mapstone, Madalina Tivarus, Eric Miller, and Jianhui Zhong are responsible for design of the work and interpretation of data for the work, revising it critically for important intellectual content, final approval and accountability of the version to be published. Miriam Weber and Roberto Arduino are responsible for interpretation of data for the work, revising it critically for important intellectual content, final approval, and accountability of the version to be published. Giovanni Schifitto is responsible for conception and design of the work, interpretation of data for the work, drafting the work, revising it critically, and final approval and accountability of the version to be published.

\section{Compliance with ethical standards}

Conflict of interest Eric Miller is the author of the CalCAP Reaction Time software used in this study.

Source of funding Giovanni SCHIFITTO received the following grants: R01 MH099921, R01 HL123346, and UL1TR002001. Xing QIU and Lu WANG received a P30 AI078498-08 grant.

Open Access This article is distributed under the terms of the Creative Commons Attribution 4.0 International License (http:// creativecommons.org/licenses/by/4.0/), which permits unrestricted use, distribution, and reproduction in any medium, provided you give appropriate credit to the original author(s) and the source, provide a link to the Creative Commons license, and indicate if changes were made.

\section{References}

Antinori A, Arendt G, Becker JT, Brew BJ, Byrd DA, Cherner M, Clifford DB, Cinque P, Epstein LG, Goodkin K, Gisslen M, Grant I, Heaton RK, Joseph J, Marder K, Marra CM, McArthur JC, Nunn M, Price RW, Pulliam L, Robertson KR, Sacktor N, Valcour V, Wojna VE (2007) Updated research nosology for HIV-associated neurocognitive disorders. Neurology 69(18):1789-1799
Beal SL (2001) Ways to fit a PK model with some data below the quantification limit. J Pharmacokinet Pharmacodyn 28(5):481-504

Becker KM, Heinrichs-Graham E, Fox HS, Robertson KR, Sandkovsky U, O'Neill J, Swindells S, Wilson TW (2013) Decreased MEG beta oscillations in HIV-infected older adults during the resting state. J Neuro-Oncol 19(6):586-594. doi:10.1007/s13365-013-0220-8

Bell AJ, Sejnowski TJ (1995) An information maximization approach to blind separation and blind deconvolution. Neural Comput 7(6): 1129-1159. doi:10.1162/neco.1995.7.6.1129

Best BM, Letendre SL, Koopmans P, Rossi SS, Clifford DB, Collier AC, Gelman BB, Marra CM, McArthur JC, McCutchan JA, Morgello S, Simpson DM, Capparelli EV, Ellis RJ, Grant I, Group CS (2012) Low cerebrospinal fluid concentrations of the nucleotide HIV reverse transcriptase inhibitor, tenofovir. J Acquir Immune Defic Syndr 59(4):376-381. doi:10.1097/QAI.0b013e318247ec54

Calcagno A, Bonora S, Simiele M, Rostagno R, Tettoni MC, Bonasso M, Romito A, Imperiale D, D'Avolio A, Di Perri G (2011) Tenofovir and emtricitabine cerebrospinal fluid-to-plasma ratios correlate to the extent of blood-brainbarrier damage. AIDS 25(11):1437-1439. doi:10.1097/QAD.0b013e3283489cb1

Calhoun VD, Adali T, Pearlson GD, Pekar JJ (2001) A method for making group inferences from functional MRI data using independent component analysis. Hum Brain Mapp 14(3):140-151. doi:10.1002/ hbm. 1048

Chang L, Ernst T, Leonido-Yee M, Witt M, Speck O, Walot I, Miller EN (1999) Highly active antiretroviral therapy reverses brain metabolite abnormalities in mild HIV dementia. Neurology 53(4):782-789

Chang L, Yakupov R, Nakama H, Stokes B, Ernst T (2008) Antiretroviral treatment is associated with increased attentional load-dependent brain activation in HIV patients. J Neuroimmune Pharmacol 3(2): 95-104. doi:10.1007/s11481-007-9092-0

Chao-Gan Y, Yu-Feng Z (2010) DPARSF: a MATLAB toolbox for "pipeline" data analysis of resting-state fMRI. Front Syst Neurosci 4:13. doi: $10.3389 /$ fnsys.2010.00013

Cysique LA, Vaida F, Letendre S, Gibson S, Cherner M, Woods SP, McCutchan JA, Heaton RK, Ellis RJ (2009) Dynamics of cognitive change in impaired HIV-positive patients initiating antiretroviral therapy. Neurology 73:342-348

Delahunty T, Bushman L, Robbins B, Fletcher CV (2009) The simultaneous assay of tenofovir and emtricitabine in plasma using LC/MS/ MS and isotopically labeled internal standards. J Chromatogr B Analyt Technol Biomed Life Sci 877(20-21):1907-1914. doi:10. 1016/j.jchromb.2009.05.029

Dietrich O, Reiser MF, Schoenberg SO (2008) Artifacts in 3-T MRI: physical background and reduction strategies. Eur J Radiol 65(1): 29-35. doi:10.1016/j.ejrad.2007.11.005

Erhardt EB, Rachakonda S, Bedrick EJ, Allen EA, Adali T, Calhoun VD (2011) Comparison of multi-subject ICA methods for analysis of fMRI data. Hum Brain Mapp 32(12):2075-2095. doi:10.1002/ hbm. 21170

Greve DN, Fischl B (2009) Accurate and robust brain image alignment using boundary-based registration. NeuroImage 48(1):63-72. doi: 10.1016/j.neuroimage.2009.06.060

Herting MM, Uban KA, Williams PL, Gautam P, Huo Y, Malee K, Yogev R, Csernansky J, Wang L, Nichols S, Van Dyke R, Sowell ER (2015) Default mode connectivity in youth with perinatally acquired HIV. Medicine (Baltimore) 94(37):e1417. doi:10.1097/MD. 0000000000001417

Hua X, Boyle CP, Harezlak J, Tate DF, Yiannoutsos CT, Cohen R, Schifitto G, Gongvatana A, Zhong J, Zhu T, Taylor MJ, Campbell TB, Daar ES, Alger JR, Singer E, Buchthal S, Toga AW, Navia B, Thompson PM, Consortium HIVN (2013) Disrupted cerebral metabolite levels and lower nadir CD4 + counts are linked to brain volume deficits in $210 \mathrm{HIV}$-infected patients on stable treatment. Neuroimage Clin 3:132-142. doi:10.1016/j.nicl.2013.07.009 
Ipser JC, Brown GG, Bischoff-Grethe A, Connolly CG, Ellis RJ, Heaton RK, Grant I, Translational Methamphetamine ARCG (2015) HIV infection is associated with attenuated frontostriatal intrinsic connectivity: a preliminary study. J Int Neuropsychol Soc 21(3):203-213. doi:10.1017/S1355617715000156

Janssen MA, Hinne M, Janssen RJ, van Gerven MA, Steens SC, Goraj B, Koopmans PP, Kessels RP (2016) Resting-state subcortical functional connectivity in HIV-infected patients on long-term cART. Brain Imaging Behav. doi:10.1007/s11682-016-9632-4

Jenkinson M, Beckmann CF, Behrens TE, Woolrich MW, Smith SM (2012) FSL Neuroimage 62(2):782-790. doi:10.1016/j. neuroimage.2011.09.015

Joel D, Berman Z, Tavor I, Wexler N, Gaber O, Stein Y, Shefi N, Pool J, Urchs S, Margulies DS, Liem F, Hanggi J, Jancke L, Assaf Y (2015) Sex beyond the genitalia: the human brain mosaic. Proc Natl Acad Sci U S A 112(50):15468-15473. doi:10.1073/pnas.1509654112

Ma Q, Liu X, Heaton R ZF, Jin H, Wu H, Crescini M, Zhao H, Letendre S (2015) Neurocognitive decline is associated with antiretroviral concentrations in cerebral spinal fluid and blood 2015;CROI Seattle, Feb 23-26 Poster 444

Marra CM, Zhao Y, Clifford DB, Letendre S, Evans S, Henry K, Ellis RJ, Rodriguez B, Coombs RW, Schifitto G, McArthur JC, Robertson K (2009) Impact of combination antiretroviral therapy on cerebrospinal fluid HIV RNA and neurocognitive performance. AIDS (London, England) 23:1359-1366

Navia BA, Cho ES, Petito CK, Price RW (1986) The AIDS dementia complex: II. Neuropathol Ann Neurol 19:525-535

Nichols TE, Holmes AP (2002) Nonparametric permutation tests for functional neuroimaging: a primer with examples. Hum Brain Mapp 15(1):1-25

Ortega M, Brier MR, Ances BM (2015) Effects of HIV and combination antiretroviral therapy on cortico-striatal functional connectivity. AIDS (London, England) 29(6):703-712. doi:10.1097/qad. 0000000000000611

Pfefferbaum A, Rosenbloom MJ, Adalsteinsson E, Sullivan EV (2007) Diffusion tensor imaging with quantitative fibre tracking in HIV infection and alcoholism comorbidity: synergistic white matter damage. Brain 130(Pt 1):48-64

Pomara N, Crandall DT, Choi SJ, Johnson G, Lim KO (2001) White matter abnormalities in HIV-1 infection: a diffusion tensor imaging study. Psychiatry Res 106(1):15-24

Ragin AB, Wu Y, Storey P, Cohen BA, Edelman RR, Epstein LG (2005) Diffusion tensor imaging of subcortical brain injury in patients infected with human immunodeficiency virus. J Neuro-Oncol 11(3): 292-298

Ragin AB, Wu Y, Gao Y, Keating S, Du H, Sammet C, Kettering CS, Epstein LG (2015) Brain alterations within the first 100 days of HIV infection. Ann Clin Translat Neurol 2(1):12-21. doi:10.1002/acn3. 136

Robertson KR, Su Z, Margolis DM, Krambrink A, Havlir DV, Evans S, Skiest DJ (2010) Neurocognitive effects of treatment interruption in stable HIV-positive patients in an observational cohort. Neurology 74(16):1260-1266

Sax PE, Tierney C, Collier AC, Fischl MA, Mollan K, Peeples L, Godfrey C, Jahed NC, Myers L, Katzenstein D, Farajallah A, Rooney JF, Ha B, Woodward WC, Koletar SL, Johnson VA, Geiseler PJ, Daar ES, Team ACTGSA (2009) Abacavirlamivudine versus tenofovir-emtricitabine for initial HIV-1 therapy. N Engl J Med 361(23):2230-2240. doi:10.1056/NEJMoa0906768
Smith SM (2002) Fast robust automated brain extraction. Hum Brain Mapp 17(3):143-155. doi:10.1002/hbm.10062

Smith SM, Nichols TE (2009) Threshold-free cluster enhancement: addressing problems of smoothing, threshold dependence and localisation in cluster inference. NeuroImage 44(1):83-98. doi:10. 1016/j.neuroimage.2008.03.061

Smith SM, Jenkinson M, Woolrich MW, Beckmann CF, Behrens TE, Johansen-Berg H, Bannister PR, De Luca M, Drobnjak I, Flitney DE, Niazy RK, Saunders J, Vickers J, Zhang Y, De Stefano N, Brady JM, Matthews PM (2004) Advances in functional and structural MR image analysis and implementation as FSL. NeuroImage 23(Suppl 1):S208-S219. doi:10.1016/j.neuroimage.2004.07.051

Smith SM, Jenkinson M, Johansen-Berg H, Rueckert D, Nichols TE, Mackay CE, Watkins KE, Ciccarelli O, Cader MZ, Matthews PM, Behrens TE (2006) Tract-based spatial statistics: voxelwise analysis of multi-subject diffusion data. NeuroImage 31(4):1487-1505. doi: 10.1016/j.neuroimage.2006.02.024

Smurzynski M, Wu K, Letendre S, Robertson K, Bosch RJ, Clifford DB, Evans S, Collier AC, Taylor M, Ellis R (2011) Effects of central nervous system antiretroviral penetration on cognitive functioning in the ALLRT cohort. AIDS (London, England) 25(3):357-365

Supekar K, Uddin LQ, Prater K, Amin H, Greicius MD, Menon V (2010) Development of functional and structural connectivity within the default mode network in young children. NeuroImage 52(1):290 301. doi:10.1016/j.neuroimage.2010.04.009

Thomas JB, Brier MR, Snyder AZ, Vaida FF, Ances BM (2013) Pathways to neurodegeneration: effects of HIV and aging on resting-state functional connectivity. Neurology 80(13):11861193. doi:10.1212/WNL.0b013e318288792b

Tozzi V, Balestra P, Lorenzini P, Bellagamba R, Galgani S, Corpolongo A, Vlassi C, Larussa D, Zaccarelli M, Noto P, Visco-Comandini U, Giulianelli M, Ippolito G, Antinori A, Narciso P (2005) Prevalence and risk factors for human immunodeficiency virus\&ndash; associated neurocognitive impairment, 1996 to 2002: results from an urban observational cohort. J Neurovirol 11(3):265-273

Valade E, Treluyer JM, Bouazza N, Ghosn J, Foissac F, Benaboud S, Fauchet F, Viard JP, Urien S, Hirt D (2014) Population pharmacokinetics of emtricitabine in HIV-1-infected adult patients. Antimicrob Agents Chemother 58(4):2256-2261. doi:10.1128/ AAC.02058-13

Wang X, Foryt P, Ochs R, Chung JH, Wu Y, Parrish T, Ragin AB (2011) Abnormalities in resting-state functional connectivity in early human immunodeficiency virus infection. Brain Connect 1(3):207217. doi:10.1089/brain.2011.0016

Winkler AM, Ridgway GR, Webster MA, Smith SM, Nichols TE (2014) Permutation inference for the general linear model. NeuroImage 92: 381-397. doi:10.1016/j.neuroimage.2014.01.060

Woolrich MW, Jbabdi S, Patenaude B, Chappell M, Makni S, Behrens T, Beckmann C, Jenkinson M, Smith SM (2009) Bayesian analysis of neuroimaging data in FSL. NeuroImage 45(1 Suppl):S173-S186. doi:10.1016/j.neuroimage.2008.10.055

Wu YC, Field AS, Whalen PJ, Alexander AL (2011) Age- and genderrelated changes in the normal human brain using hybrid diffusion imaging (HYDI). NeuroImage 54(3):1840-1853. doi:10.1016/j. neuroimage.2010.09.067

Zhu T, Zhong J, Hu R, Tivarus M, Ekholm S, Harezlak J, Ombao H, Navia B, Cohen R, Schifitto G (2013) Patterns of white matter injury in HIV infection after partial immune reconstitution: a DTI tractbased spatial statistics study. J Neurovirol 19(1):10-23. doi:10. 1007/s13365-012-0135-9 